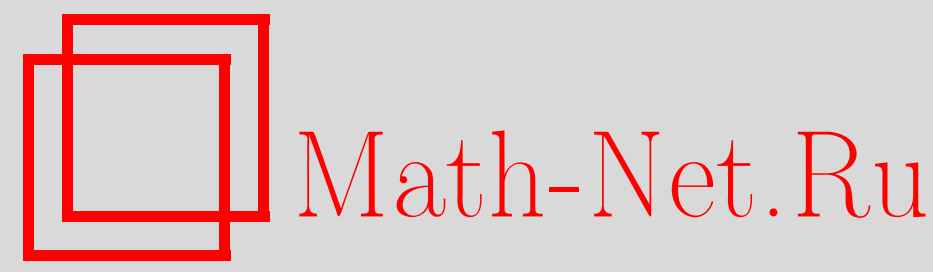

Ю. А. Неретин, Действие надалгебры в планшерелевском разложении и операторы сдвига в мнимом направлении, Изв. РАН. Сер. матем., 2002, том 66, выпуск 5, 171182

DOI: https://doi.org/10.4213/im404

Использование Общероссийского математического портала Math-Net.Ru подразумевает, что вы прочитали и согласны с пользовательским соглашением

http://www . mathnet.ru/rus/agreement

Параметры загрузки:

IP : 35.173 .137 .237

26 апреля 2023 г., 15:52:34 
УДК 519.46

\author{
Ю. А. Неретин
}

\title{
Действие надалгебры в планшерелевском разложении и операторы сдвига в мнимом направлении
}

\begin{abstract}
Рассматривается тензорное произведение унитарного представления группы $G=\mathrm{SL}_{2}(\mathbb{R})$ со старшим весом и комплексно-сопряженного представления с младшим весом. В пространстве представления действует прямое произведение групп $G \times G$. Разложим полученное представление в прямой интеграл относительно диагональной подгруппы $G \subset G \times G$, этот прямой интеграл реализуется как пространство $L^{2}$ на произведении окружности с координатой $\phi \in[0,2 \pi)$ и полупрямой $s \geqslant 0$, где $s$ нумерует унитарные представления группы $G$ основной серии.

Получены явные формулы для действия алгебры Ли $\mathfrak{s} l_{2} \oplus \mathfrak{s} l_{2}$ в этом прямом интеграле. Оказывается, что операторы представления являются дифференциальными операторами второго порядка по ф и разностными операторами второго порядка по $s$, причем разностные операторы выражаются через сдвиг в мнимом направлении $s \mapsto s+i$.

Библиография: 32 наименования.
\end{abstract}

\section{0. Введение.}

В последние 50 лет было решено много задач о явном спектральном разложении ограничений унитарных представлений на подгруппы (см. библиографию в [22]).

Этот класс задач содержит некоторые проблемы, которые обычно формулируются в других терминах.

1. Разложение тензорного произведения представлений $\rho_{1}, \rho_{2}$ группь $G$. Действительно, это в точности задача об ограничении представлений группы $G \times G$ на диагональную подгруппу $G \subset G \times G$.

2. Разложсние $L^{2}$ на псевдоримановом симметрическом пространстве $G / H$. Как было показано в [23], для любого классического псевдориманова симметрического пространства $G / H$ сушествуют классическая группа $\widetilde{G} \supset G$ и представление $\rho$ основной вырожденной серии группы $\widetilde{G}$, удовлетворяющее одному из следующих двух свойств (обычно первому):

1) ограничение $\rho$ на $G$ есть $L^{2}(G / H)$;

2 ) ограничение $\rho$ на $G$ есть прямая сумма пространств $L^{2}\left(G / H_{j}\right)$, где $G / H_{j}-$ конечный набор симметрических пространств, а $G / H$ - одно из пространств $G / H_{j}$.

Следовательно, разложение $L^{2}$ на классическом псевдоримановом симметрическом пространстве может рассматриваться как задача об ограничении представлений ${ }^{1}$.

Работа поддержана грантом NWO 047-008-009.

${ }^{1}$ Это явление легко увидеть из статьи Макаревича [18], опубликованной в 1973 г., странным образом оно оставалось не сформулированным в течение длительного времени.

(C) Ю.А. Неретин, 2002 
Описание спектрального типа (без явной формулы Планшереля) для всех псевдоримановых симметрических пространств было недавно получено в работах ван ден Бана, Шлихткрулля, Делорма и Ошимы (доказательство содержится в объединении большого числа работ, ссылки см. в [2], [7]). По-видимому, для классических симметрических пространств проблема явного вычисления меры Планшереля близка к полному решению.

Для некоторых случаев явная мера Планшереля известна; в частности, для $L^{2}$ на полупростых группах (см. [9], [14]), на римановых симметрических пространствах (см. [9]-[11], а также [15]), на пространствах ранга 1 (см. [20], [21]) и на пространствах $G_{\mathbb{C}} / G_{\mathbb{R}}$, где $G_{\mathbb{C}}$ - комплексная группа, а $G_{\mathbb{R}}$ - ее вещественная форма (см. [12], [13]).

3. Керн-представления Березина (деформация $L^{2}$ на псевдоримановом симметрическом пространстве $G / K$ ) также могут быть получены как ограничения с некоторой надгруппы $G^{0} \supset G$ (см. [24], [26] и ссылки в [26]).

Обычно проблема некоммутативного гармонического анализа формулируется как задача о спектре представления или как более сложная задача о явном разложении представления в прямой интеграл неприводимых представлений. Последний вопрос включает явное вычисление спектральной меры (так называемой меры Планшереля). Недавние работы автора [25], [26] содержат попытку изучения "анализа после формулы Планшереля".

Настоящая работа является продолжением статьи [25]. Здесь мы пытаемся понять, возможен ли ответ на следующий вопрос.

ВоПрос. Пусть нам известна явная формула Планшереля для ограничения унитарного представления $\rho$ группь $G$ на подгруппу $H$. Возможно ли явно выписать формуль, задающие действие алгебры Ли группь $G$ в прямом интеграле представлений $H$ ?

Мы получаем положительное решение этой проблемы в одном из простейших возможных случаев, а именно для тензорного произведения представления $\operatorname{SL}(2, \mathbb{R})$ со старшим весом и комплексно-сопряженного представления с младшим весом. Это тензорное произведение и его разложение многократно рассматривались в литературе по теории представлений и специальным функциям в последние 40 лет (см., например, [27], [5], [31], [19], [8], [28], [29]). Однако формулы для действия алгебры Ли $\mathrm{sl}_{2} \oplus \mathrm{sl}_{2}$ в тензорном произведении не были известны. Причина - необычный для теории представлений вид этих операторов.

Оказывается, что выписанные ниже операторы (12)-(14) алгебры Ли $\mathrm{sl}_{2} \oplus \mathrm{sl}_{2}$ являются дифференциальными операторами второго порядка по одной переменной и разностными операторами второго порядка по другой переменной; более того, оказывается, что разностные операторы определены через сдвиг в мнимом направлении, т. е. в нашей формуле появляются операторы вида

$$
T f(x)=f(x+i), \quad f \in L^{2}(\mathbb{R})
$$

$\left(i^{2}=-1\right)$. Здесь нет внутреннего противоречия, разностные операторы корректно определены на функциях, допускающих аналитическое продолжение в подходящую полосу. Ведь стандартные операторы $f \mapsto x f$ и $f \mapsto \frac{d}{d x} f$ тоже не определены во всем пространстве $L^{2}(\mathbb{R})$. 
Сначала эти операторы были получены автором с помощью теорем об операционном исчислении для индексного гипергеометрического преобразования (см. ниже формулу (22)) из [25]. Но окончательные формулы оказываются элементарными и могут быть проверены непосредственно элементарными методами.

Так как примерная структура формул для действия надалгебры теперь стала относительно понятной, естественно сформулировать более общую задачу, что и сделано выше.

Настояшая работа была выполнена во время моего пребывания в институте им. Эрвина Шрёдингера (Вена). Я благодарю администрацию института за гостеприимство.

1. Группа $\mathrm{SL}(2, \mathbb{R})$. Зададим группу $\mathrm{SL}(2, \mathbb{R})$ как группу комплексных $(2 \times 2)$ матрищ вида

$$
\left(\begin{array}{cc}
a & b \\
\bar{b} & \bar{a}
\end{array}\right), \quad|a|^{2}-|b|^{2}=1
$$

Через $D$ обозначим круг $|z|<1$ на комплексной плоскости $\mathbb{C}$, а через $S^{1}-$ окружность $|z|=1$. Представим точки окружности в виде $z=e^{i \varphi}$.

Группа $\mathrm{SL}(2, \mathbb{R})$ действует на круге $D$ мёбиусовскими преобразованиями

$$
\left(\begin{array}{cc}
a & b \\
\bar{b} & \bar{a}
\end{array}\right): \quad z \mapsto \frac{a z+b}{\bar{b} z+\bar{a}}
$$

Если точка $z \in S^{1}$, то ее образ под действием (1) также содержится в $S^{1}$.

2. Представления группы $\mathrm{SL}(2, \mathbb{R})$ со старшим весом. Фиксируем $\alpha>1$. Рассмотрим пространство $H_{\alpha}$ голоморфных в круге $D$ функций $f$, удовлетворяющих условию

$$
\int_{D}|f(z)|^{2}\left(1-|z|^{2}\right)^{\alpha-2}\{d z\}<\infty,
$$

где $\{d z\}$ обозначает меру Лебега на $D$. Определим скалярное произведение в пространстве $H_{\alpha}$ по формуле

$$
\langle f, g\rangle_{\alpha}=\frac{\alpha-1}{\pi} \int_{D} f(z) \overline{g(z)}\left(1-|z|^{2}\right)^{\alpha-2}\{d z\} ;
$$

предьнтегральный множитель обеспечивает равенство $\langle 1,1\rangle_{\alpha}=1$. Выражение скалярного произведения через коэффициенты Тейлора имеет вид

$$
\left\langle\sum c_{k} z^{k}, \sum c_{k}^{\prime} z^{k}\right\rangle_{\alpha}=\sum_{k \geqslant 0} c_{k} \bar{c}_{k}^{\prime} \frac{k !}{\alpha(\alpha+1) \ldots(\alpha+k-1)}
$$

а условие (2) для $f(z)=\sum c_{k} z^{k}$ переписывается как

$$
\sum\left|c_{k}\right|^{2} k^{-(\alpha-1)}<\infty
$$

Легко проверить, что пространство $H_{\alpha}$ полно по отношению к норме, заданной этим скалярным произведением, т. е. $H_{\alpha}$ - гильбертово пространство. 
Группа $\mathrm{SL}(2, \mathbb{R})$ действует в $H_{\alpha}$ унитарными операторами

$$
T_{\alpha}\left(\begin{array}{cc}
a & b \\
\bar{b} & \bar{a}
\end{array}\right)(z)=f\left(\frac{a z+b}{\bar{b} z+\bar{a}}\right)(\bar{b} z+\bar{a})^{-\alpha} .
$$

Рассмотрим сначала случай, когда число $\alpha$ целое. Множитель $(\bar{b} z+\bar{a})^{-\alpha}$ является степенью производной функции (1), поэтому $T_{\alpha}$ является представлением:

$$
T_{\alpha}\left(g_{1}\right) T_{\alpha}\left(g_{2}\right)=T_{\alpha}\left(g_{1} g_{2}\right) .
$$

Операторы $T_{\alpha}(g)$ унитарны, т. е.

$$
\left\langle T_{\alpha}(g) f_{1}, T_{\alpha}(g) f_{2}\right\rangle_{\alpha}=\left\langle f_{1}, f_{2}\right\rangle_{\alpha}
$$

это легко проверить, заменяя переменную в интеграле.

Если $\alpha$ не целое, то $T_{\alpha}(g)$ также является унитарным представлением; мы должны лишь объяснить смысл выражения

$$
(\bar{b} z+\bar{a})^{-\alpha}=\left(1+b \bar{a}^{-1} z\right)^{-\alpha} a^{-\alpha}=\left(1+b \bar{a}^{-1} z\right)^{-\alpha} e^{-\alpha(\ln a+2 \pi i k)} .
$$

Очевидно, $\left|b \bar{a}^{-1}\right|<1$. Следовательно, функция

$$
\left(1+b \bar{a}^{-1} z\right)^{-\alpha}:=1+\frac{\alpha}{1 !} b \bar{a}^{-1} z+\frac{\alpha(\alpha-1)}{2 !}\left(b \bar{a}^{-1}\right)^{2} z^{2}+\cdots
$$

корректно определена. Поэтому выражение (5) определено с точностью до множителя $e^{-2 \pi i k \alpha}$, по модулю равного 1 . Следовательно, равенство (6) заменяется на равенство

$$
T_{\alpha}\left(g_{1}\right) T_{\alpha}\left(g_{2}\right)=\theta \cdot T_{\alpha}\left(g_{1} g_{2}\right),
$$

где $|\theta|=1$. Итак, $T_{\alpha}(g)$ - унитарное проективное представление группы $\operatorname{SL}(2, \mathbb{R})$.

ЗАмЕчАниЕ. Очевидно, $T_{\alpha}(g)$ может также рассматриваться как линейное представление универсальной накрываюшей группы $\mathrm{SL}(2, \mathbb{R})$.

3. Тензорное произведение. Через $\bar{T}_{\alpha}(g)$ обозначим представление, комплексно-сопряженное к $T_{\alpha}(g)$, оно действует в пространстве $\bar{H}_{\alpha}$ антиголоморфных функций в круге $|u|<1$ по формуле

$$
T_{\alpha}\left(\begin{array}{cc}
a & b \\
\bar{b} & \bar{a}
\end{array}\right) f(\bar{u})=f\left(\frac{\bar{a} \bar{u}+\bar{b}}{b \bar{u}+a}\right)(b \bar{u}+a)^{-\alpha} .
$$

Скалярное произведение в $\bar{H}_{\alpha}$ задается той же формулой (3).

Рассмотрим тензорное произведение $H_{\alpha} \otimes \bar{H}_{\alpha}$. Оно состоит из функций $f(z, \bar{u})$ на бикруге $D \times D$, голоморфных по $z$ и антиголоморфных по $u$; скалярное произведение в $H_{\alpha} \otimes \bar{H}_{\alpha}$ имеет вид

$$
\left\langle f_{1}, f_{2}\right\rangle=\frac{(\alpha-1)^{2}}{\pi^{2}} \iint_{D \times D} f_{1}(z, \bar{u}) \overline{f_{2}(z, \bar{u})}(1-z \bar{z})^{\alpha-2}(1-u \bar{u})^{\alpha-2}\{d z\}\{d u\} .
$$


Группа $\mathrm{SL}_{2}(\mathbb{R}) \times \mathrm{SL}_{2}(\mathbb{R})$ действует в $H_{\alpha} \otimes \bar{H}_{\alpha}$ операторами $T_{\alpha}\left(g_{1}\right) \otimes \bar{T}_{\alpha}\left(g_{2}\right)$ вида

$$
\begin{aligned}
T_{\alpha} & \left(\begin{array}{cc}
a_{1} & b_{1} \\
\bar{b}_{1} & \bar{a}_{1}
\end{array}\right) \otimes \bar{T}_{\alpha}\left(\begin{array}{cc}
a_{2} & b_{2} \\
\bar{b}_{2} & \bar{a}_{2}
\end{array}\right) f(z, u) \\
& =f\left(\frac{a_{1} z+b_{1}}{\bar{b}_{1} z+\bar{a}_{1}}, \frac{\bar{a}_{2} \bar{u}+\bar{b}_{2}}{b_{2} \bar{u}+a_{2}}\right)\left(\bar{b}_{1} z+\bar{a}_{1}\right)^{-\alpha}\left(b_{2} \bar{u}+a_{2}\right)^{-\alpha} .
\end{aligned}
$$

Ограничим это представление на диагональную подгруппу $\mathrm{SL}_{2}(\mathbb{R}) \subset \mathrm{SL}_{2}(\mathbb{R}) \times$ $\mathrm{SL}_{2}(\mathbb{R})$, это соответствует подстановке $a_{1}=a_{2}=a, b_{1}=b_{2}=b$ в последнюю формулу. Полученное представление групшы $\mathrm{SL}_{2}(\mathbb{R})$ является линейным (не проективньгм). Действительно,

$$
(\bar{b} z+\bar{a})^{-\alpha}(b \bar{u}+a)^{-\alpha}=\left(1+\bar{a}^{-1} \bar{b} z\right)^{-\alpha}\left(1+a^{-1} b \bar{u}\right)^{-\alpha}(a \bar{a})^{-\alpha},
$$

и это выражение является однозначной функцией при $|z|<1,|u|<1$.

4. Основная серия представлений. Фиксируем $s \in \mathbb{R}$. Рассмотрим представление $\rho_{s}$ групшы $\mathrm{SL}(2, \mathbb{R})$, действуюшее в $L^{2}$ на круге $S^{1}$ по формуле

$$
\rho_{s}\left(\begin{array}{ll}
a & b \\
\bar{b} & \bar{a}
\end{array}\right) f\left(e^{i \varphi}\right)=f\left(\frac{a e^{i \varphi}+b}{\bar{b} e^{i \varphi}+\bar{a}}\right)\left|\bar{b} e^{i \varphi}+\bar{a}\right|^{-1-2 i s} .
$$

Это так называемые представления основной серии. Напомним, что представление $T_{s}$ эквивалентно $T_{-s}$ (см. [3]).

5. Спектральное разложение. Пусть $\alpha$-то же, что и выше. Рассмотрим ядро

$$
K_{\alpha}(\varphi, s ; z, u):=\frac{\left(1-\bar{z} e^{i \varphi}\right)^{-1 / 2-i s}\left(1-u e^{-i \varphi}\right)^{-1 / 2-i s}}{(1-\bar{z} u)^{\alpha-1 / 2-i s}},
$$

где $|z|<1,|u|<1, \varphi \in[0,2 \pi], s \in \mathbb{R}$. Рассмотрим интегральный оператор $J_{\alpha}$, переводящий функцию $f \in H_{\alpha} \otimes \bar{H}_{\alpha}$ в функцию $F(\varphi, s)$, заданную формулой

$$
F(\varphi, s)=\iint_{D \times D} K_{\alpha}(\varphi, s ; z, u) f(z, \bar{u})(1-z \bar{z})^{\alpha-2}(1-u \bar{u})^{\alpha-2}\{d z\}\{d u\} .
$$

Рассмотрим действие группы $\mathrm{SL}(2, \mathbb{R})$ в пространстве функций от переменных $(\varphi, s)$, определенное формулой

$$
R\left(\begin{array}{cc}
a & b \\
\bar{b} & \bar{a}
\end{array}\right) F\left(e^{i \varphi}, s\right)=F\left(\frac{a e^{i \varphi}+b}{\bar{b} e^{i \varphi}+\bar{a}}, s\right)\left|\bar{b} e^{i \varphi}+\bar{a}\right|^{-1-2 i s}
$$

Заметим, что при фиксированном $s \in \mathbb{R}$ функция $F\left(e^{i \varphi}, s\right)$ (как функция от $\varphi$ ) преобразуется по формуле для основной серии.

Простое вычисление показывает, что оператор $J_{\alpha}$ сплетает представления $T_{\alpha}(g) \otimes \bar{T}_{\alpha}(g)$ и $R$ групшы $\mathrm{SL}(2, \mathbb{R}):$

$$
J_{\alpha} \cdot\left(T_{\alpha}(g) \otimes \bar{T}_{\alpha}(g)\right)=R(g) \cdot J_{\alpha} .
$$


TЕОРема 1. Оператор $J_{\alpha}$ является унитарным оператором из $H_{\alpha} \otimes \bar{H}_{\alpha}$ в $L^{2}$ на полосе $0 \leqslant \varphi \leqslant 2 \pi, s \geqslant 0$ по мере

$$
\frac{|\Gamma(\alpha-1 / 2+i s)|^{2}}{\Gamma(\alpha)^{2}} s \frac{\operatorname{sh}(\pi s)}{\operatorname{ch}(\pi s)} d s d \varphi=\left|\frac{\Gamma(\alpha-1 / 2+i s) \Gamma(1 / 2+i s)}{\Gamma(\alpha) \Gamma(i s)}\right|^{2} d s d \varphi .
$$

Итак, $T_{\alpha} \otimes \bar{T}_{\alpha}$ является однократньм прямым интегралом по основной серии представлений (этот факт был обнаружен Пуканским [27]). Различные способы получения меры Планшереля (10) можно найти в [31], [19], [8], [25].

6. Голоморфное продолжение $J_{\alpha} f(\varphi, s)$. Обозначим через $W$ пространство всех функций $f \in H_{\alpha} \otimes \bar{H}_{\alpha}$, гладких вплоть до гранищы бидиска $|z|<1,|u|<1$. Пусть $f \in W$. Рассмотрим ее ряд Тейлора

$$
f(z, \bar{u})=\sum_{k, l} c_{k l} z^{k} \bar{u}^{l} .
$$

Очевидно, коэффициенты $c_{k l}$ быстро убывают при $k+l \rightarrow \infty$, т. е. для всех $N$

$$
\left|c_{k, l}\right|=o(k+l)^{-N} .
$$

Лемма. Фиксируем $\varphi$. Для любой $f \in W$ функщия $J_{\alpha} f(\varphi, s)$ продолжается голоморфно по $s$ во всю комплексную плоскость $\mathbb{C}$.

ДокаЗАтЕльСТво. Фиксируем $s \in \mathbb{C}, \varphi \in[0,2 \pi]$. Функция $K_{\alpha}(\varphi, s ; z, u)$ как функция переменных $z, u$ имеет полиномиальный рост при подходе к гранище бидиска $D \times D$ :

$\left|K_{\alpha}(\varphi, s ; z, u)\right| \leqslant \exp \{4 \pi(1+|\operatorname{Im} s|)\}(1-|z|)^{-(1+\alpha+2|\operatorname{Re} s|)}(1-|u|)^{-(1+\alpha+2|\operatorname{Re} s|)}$.

Поэтому для фиксированных $s, \varphi$ коэффициенты $a_{k l}=a_{k l}(\varphi, s)$ ряда

$$
K_{\alpha}(\varphi, s ; z, u)=\sum a_{k l}(s, \varphi) \bar{z}^{k} u^{l}
$$

имеют полиномиальньй рост при $k, l \rightarrow+\infty$.

Действительно, рассмотрим функцию $q(z, u)$ в бидиске, удовлетворяющую

$$
|q(z, u)| \leqslant C \cdot \delta^{-h}, \quad|z| \leqslant 1-\delta, \quad|u| \leqslant 1-\delta .
$$

Тейлоровские коэффициенты $b_{k l}$ функции $q(z, u)$ задаются формулой

$$
b_{k l}=\frac{1}{(2 \pi i)^{2}} \iint_{|z|=1-\delta,|u|=1-\delta} \frac{q(z, \bar{u}) d z d u}{z^{k+1} \bar{u}^{l+1}} .
$$

Поэтому для некоторой константы $C$ выполнено

$$
\left|b_{k l}\right| \leqslant C \delta^{-h}(1-\delta)^{-k-l-2}
$$

для всех $\delta$. Мы выбираем $\delta=h /(h+k+l+2)$ и получаем полиномиальный рост для коэффициентов $b_{k l}$.

Для ядра $K_{\alpha}(z, u)$ таким способом получаем оценки вида $\left|a_{k l}\right| \leqslant C(1+k+l)^{\tau}$ в каждом прямоугольнике

$$
|\operatorname{Im} s| \leqslant N, \quad|\operatorname{Re} s| \leqslant M .
$$


Так как тейлоровские коэффициенты $c_{k l}$ для $f \in W$ быстро убывают, ряд

$$
J_{\alpha} f(\varphi, s)=\sum c_{k l} a_{k l}(s, \varphi) \frac{k ! l !}{(\alpha)_{k}(\alpha)_{l}}
$$

абсолютно сходится (см. (4)); слагаемые голоморфны по $s$, и ряд равномерно сходится в прямоугольнике (11).

7. Соответствие дифференциальных операторов. Операторы алгебры Ли $\operatorname{sl}(2) \oplus \operatorname{sl}(2)$ в пространстве $H_{\alpha} \otimes \bar{H}_{\alpha}$ имеют вид

$$
\begin{aligned}
& L_{0}^{(z)}=z \frac{\partial}{\partial z}+\frac{\alpha}{2}, \quad L_{1}^{(z)}=z^{2} \frac{\partial}{\partial z}+\alpha z, \quad L_{-1}^{(z)}=\frac{\partial}{\partial z}, \\
& L_{0}^{(u)}=\bar{u} \frac{\partial}{\partial \bar{u}}+\frac{\alpha}{2}, \quad L_{1}^{(u)}=\frac{\partial}{\partial \bar{u}}, \quad L_{-1}^{(u)}=\bar{u}^{2} \frac{\partial}{\partial \bar{u}}+\alpha \bar{u} .
\end{aligned}
$$

Будет удобнее работать со следующим набором операторов:

$$
\begin{array}{cc}
L_{0}:=L_{0}^{(z)}-L_{0}^{(u)}, \quad L_{-1}:=L_{1}^{(u)}-L_{-1}^{(z)}, \quad L_{1}:=L_{1}^{(z)}-L_{-1}^{(u)}, \\
M_{0}:=L_{0}^{(z)}+L_{0}^{(u)}, \quad M_{-1}:=L_{1}^{(u)}+L_{-1}^{(z)}, \quad M_{1}:=L_{1}^{(z)}+L_{-1}^{(u)}
\end{array}
$$

Операторы $L_{0}, L_{1}, L_{-1}$ порождают диагональную подалгебру $\mathrm{sl}_{2} \mathrm{~B} \mathrm{sl}_{2} \oplus \mathrm{sl}_{2}$. Оператор $J_{\alpha}$ преобразует их в следуюшие операторы:

$$
\begin{aligned}
J_{\alpha} \circ\left[z \frac{\partial}{\partial z}-\bar{u} \frac{\partial}{\partial \bar{u}}\right] f(\varphi, s) & =\left[\frac{\partial}{i \partial \varphi} \circ J_{\alpha}\right] f(\varphi, s) \\
J_{\alpha} \circ\left[\bar{u}^{2} \frac{\partial}{\partial \bar{u}}+\alpha \bar{u}-\frac{\partial}{\partial z}\right] f(\varphi, s) & =-\left[e^{i \varphi} \frac{\partial}{i \partial \varphi}+\left(\frac{1}{2}+i s\right) e^{i \varphi}\right] J_{\alpha} f(\varphi, s), \\
J_{\alpha} \circ\left[z^{2} \frac{\partial}{\partial z}+\alpha z-\frac{\partial}{\partial \bar{u}}\right] & =\left[e^{-i \varphi} \frac{\partial}{i \partial \varphi}-\left(\frac{1}{2}+i s\right) e^{-i \varphi}\right] J_{\alpha} f(\varphi, s) .
\end{aligned}
$$

Эти три формулы легко следуют из (9).

ТЕОрема 2. Унитарный оператор $J_{\alpha}$ преобразует оператор $M_{0}$ в операmop

$$
\begin{aligned}
& Q_{0} f(\varphi, s)=-\frac{\left(-\frac{1}{2}+i s\right)\left(-\alpha+\frac{1}{2}+i s\right)}{2 i s} f(\varphi, s+i) \\
& \quad+\frac{\left(\frac{1}{2}+i s\right)\left(\alpha-\frac{1}{2}+i s\right)}{2 i s} f(\varphi, s-i)-\frac{-\alpha+\frac{1}{2}+i s}{2 i s\left(-\frac{1}{2}+i s\right)} \frac{\partial^{2}}{\partial \varphi^{2}} f(\varphi, s+i),
\end{aligned}
$$

m. е. для любой функиии $f \in W$ (cм. n. 6)

$$
Q_{0} J_{\alpha} f=J_{\alpha} M_{0} f
$$


Oператор $M_{1}$ переводится в оператор

$$
\begin{aligned}
Q_{1} f(\varphi, s)= & e^{i \varphi}\left[\frac{\left(\frac{1}{2}+i s\right)\left(-\alpha+\frac{1}{2}+i s\right)}{2 i s} f(\varphi, s+i)\right. \\
& +\frac{\left(\frac{1}{2}+i s\right)\left(\alpha-\frac{1}{2}+i s\right)}{2 i s} f(\varphi, s-i) \\
& \left.-\frac{-\alpha+\frac{1}{2}+i s}{2 i s\left(-\frac{1}{2}+i s\right)} \frac{d^{2}}{d \varphi^{2}} f(\varphi, s+i)+\frac{-\alpha+\frac{1}{2}+i s}{-\frac{1}{2}+i s} \frac{\partial}{i \partial \varphi} f(\varphi, s+i)\right] .
\end{aligned}
$$

Onepamop $M_{-1}$ nереходит в оператор

$$
\begin{aligned}
& Q_{-1} f(\varphi, s)=e^{-i \varphi}\left[-\frac{\left(\frac{1}{2}+i s\right)\left(-\alpha+\frac{1}{2}+i s\right)}{2 i s} f(\varphi, s+i)\right. \\
& +\frac{\left(\frac{1}{2}+i s\right)\left(\alpha-\frac{1}{2}+i s\right)}{2 i s} f(\varphi, s-i) \\
& \left.\quad-\frac{-\alpha+\frac{1}{2}+i s}{2 i s\left(-\frac{1}{2}+i s\right)} \frac{d^{2}}{d \varphi^{2}} f(\varphi, s+i)-\frac{-\alpha+\frac{1}{2}+i s}{-\frac{1}{2}+i s} \frac{\partial}{i \partial \varphi} f(\varphi, s+i)\right] .
\end{aligned}
$$

ДоКАЗАТЕЛЬСТвО. Эти формулы могут быть проверены прямым вычислением. Например, рассмотрим $M_{0}$. Очевидно, оператор $M_{0}$ самосопряжен в $H_{\alpha} \otimes \bar{H}_{\alpha}$. Поэтому

$$
\begin{array}{rl}
J_{\alpha} M_{0} & f(\varphi, s)=\iint_{D \times D} K(\varphi, s ; \bar{z}, u)\left[\left(z \frac{\partial}{\partial z}+\bar{u} \frac{\partial}{\partial \bar{u}}+\alpha\right)\right] \\
& \times f(z, \bar{u})(1-z \bar{z})^{\alpha-2}(1-u \bar{u})^{\alpha-2}\{d z\}\{d u\} \\
= & \iint_{D \times D}\left[\left(\bar{z} \frac{\partial}{\partial \bar{z}}+u \frac{\partial}{\partial u}+\alpha\right)\right] K(\varphi, s ; \bar{z}, u) \\
& \times f(z, \bar{u})(1-z \bar{z})^{\alpha-2}(1-u \bar{u})^{\alpha-2}\{d z\}\{d u\} .
\end{array}
$$

Итак, утверждение теоремы равносильно следующему:

$$
\left(u \frac{\partial}{\partial u}+\bar{z} \frac{\partial}{\partial \bar{z}}+\alpha\right) K(\varphi, s ; \bar{z}, u)-Q_{0}[K(\varphi, s ; \bar{z}, u)]=0 .
$$

После деления на $K$ это тождество принимает вид

$$
\begin{gathered}
K^{-1}\left\{u \frac{\partial}{\partial u}+z \frac{\partial}{\partial \bar{z}}\right\} K+\alpha+K^{-1} \frac{\left(-\frac{1}{2}+i s\right)\left(-\alpha+\frac{1}{2}+i s\right)}{2 i s} K(s+i) \\
-K^{-1} \frac{\left(\frac{1}{2}+i s\right)\left(\alpha-\frac{1}{2}+i s\right)}{2 i s} K(s-i) \\
+K^{-1} \frac{-\alpha+\frac{1}{2}+i s}{2 i s\left(-\frac{1}{2}+i s\right)} \frac{\partial^{2}}{\partial \varphi^{2}} K(s+i)=0
\end{gathered}
$$

Функция в левой части является длинным рациональным выражением по $z, \bar{u}, e^{i \varphi}$, $s, \alpha$; поэтому тождество (16) может быть легко проверено с помощью программы 
MAPLE. Проведем его проверку вручную. Каждое слагаемое (16) может быть представлено в виде линейной комбинации

$$
\begin{gathered}
a(s)+b(s) \\
\frac{1}{1-z \bar{u}}+c(s)\left[\frac{1}{1-z e^{i \varphi}}+\frac{1}{1-\bar{u} e^{-i \varphi}}\right] \\
+d(s) \frac{\left(1-z e^{i \varphi}\right)\left(1-\bar{u} e^{-i \varphi}\right)}{1-z \bar{u}} .
\end{gathered}
$$

После этого будет достаточно сложить коэффициенты.

Обозначим

$$
\begin{gathered}
\mu=1-z \bar{u}, \quad \nu=1-z e^{i \varphi}, \quad \theta=1-\bar{u} e^{-i \varphi}, \\
L=\frac{\left(1-z e^{i \varphi}\right)\left(1-\bar{u} e^{-i \varphi}\right)}{1-z \bar{u}} .
\end{gathered}
$$

Во-первых,

$$
\begin{aligned}
K^{-1} & \left(z \frac{\partial}{\partial z}+\bar{u} \frac{\partial}{\partial \bar{u}}+\alpha\right) K \\
& =\left(\frac{1}{2}+i s\right)\left[\frac{z e^{i \varphi}}{\mu}+\frac{\bar{u} e^{-i \varphi}}{\nu}\right]-2\left(-\alpha+\frac{1}{2}+i s\right) \frac{z \bar{u}}{\theta}+\alpha \\
& =\left(\frac{1}{2}+i s\right)\left[\frac{1}{\mu}+\frac{1}{\nu}-2\right]-2\left(-\alpha+\frac{1}{2}+i s\right)\left[\frac{1}{\theta}-1\right]+\alpha \\
& =\left(\frac{1}{2}+i s\right)\left[\frac{1}{\mu}+\frac{1}{\nu}\right]-2\left(-\alpha+\frac{1}{2}+i s\right) \frac{1}{\theta}-\alpha .
\end{aligned}
$$

Далее,

$$
\begin{aligned}
K^{-1} K(\varphi, s-i)= & \frac{1-z \bar{u}}{\left(1-z e^{i \varphi}\right)\left(1-\bar{u} e^{-i \varphi}\right)}=-1+\left[\frac{1}{\mu}+\frac{1}{\nu}\right], \\
& K^{-1} K(\varphi, s+i)=L .
\end{aligned}
$$

Наиболее громоздко последнее слагаемое в (16). Обозначим через $\mu^{\prime}, \mu^{\prime \prime}, \nu^{\prime}, \nu^{\prime \prime}$ производные от $\mu, \nu$ по $\varphi$. Легко видеть, что

$$
\frac{\partial^{2}}{\partial \varphi^{2}} K=\left(\frac{1}{2}+i s\right)\left\{\left(\frac{3}{2}+i s\right)\left[\frac{\left(\mu^{\prime}\right)^{2}}{\mu^{2}}+\frac{\left(\nu^{\prime}\right)^{2}}{\nu^{2}}\right]-\left[\frac{\mu^{\prime \prime}}{\mu}+\frac{\nu^{\prime \prime}}{\nu}\right]+2\left(\frac{1}{2}+i s\right) \frac{\mu^{\prime} \nu^{\prime}}{\mu \nu}\right\} K .
$$

Поэтому

$$
\begin{aligned}
& K^{-1} \frac{\partial^{2}}{\partial \varphi^{2}} K(\varphi, s+i)=\left(-\frac{1}{2}+i s\right) \\
& \quad \times\left\{\left(\frac{1}{2}+i s\right)\left[\frac{\left(\mu^{\prime}\right)^{2}}{\mu^{2}}+\frac{\left(\nu^{\prime}\right)^{2}}{\nu^{2}}\right]-\left[\frac{\mu^{\prime \prime}}{\mu}+\frac{\nu^{\prime \prime}}{\nu}\right]+2\left(-\frac{1}{2}+i s\right) \frac{\mu^{\prime} \nu^{\prime}}{\mu \nu}\right\} L .
\end{aligned}
$$


Далее, преобразуем все слагаемые последнего выражения:

$$
\begin{gathered}
{\left[\frac{\left(\mu^{\prime}\right)^{2}}{\mu^{2}}+\frac{\left(\nu^{\prime}\right)^{2}}{\nu^{2}}\right] L=-L+1-\left[\frac{1}{\mu}+\frac{1}{\nu}\right]+\frac{2}{\theta}} \\
{\left[\frac{\mu^{\prime \prime}}{\mu}+\frac{\nu^{\prime \prime}}{\nu}\right] L=-L+1} \\
\frac{\mu^{\prime} \nu^{\prime}}{\mu \nu} L=\frac{1}{\theta}-1 .
\end{gathered}
$$

Окончательно получаем

$$
\begin{aligned}
K^{-1} \frac{\partial^{2}}{\partial \varphi^{2}} K(\varphi, s+i)= & \left(-\frac{1}{2}+i s\right)\left\{-\left(-\frac{1}{2}+i s\right) L-\left(\frac{1}{2}+i s\right)\left[\frac{1}{\mu}+\frac{1}{\nu}\right]\right. \\
& \left.+4 i s \cdot \frac{1}{\theta}-\left(-\frac{1}{2}+i s\right)\right\} .
\end{aligned}
$$

Итак, формулы (18)-(21) содержат разложения (17) для всех слагаемых (16). Остается сложить коэффищиенты.

ЗАмечАниЕ. Применим оператор $Q_{0}$ к функциям вида $f(\varphi, s)=g(s)$. Тогда уравнение

$$
Q_{0} g(s)=(k+\alpha) g(s)
$$

становится частным случаем разностных уравнений для непрерывных двойственных многочленов Хана (continuous dual Hahn polynomials [1, (6.10.9)]).

8. Несколько замечаний. 1) Рассмотрим преобразование Меллина

$$
\hat{f}(s)=\int_{0}^{\infty} x^{i s} f(x) \frac{d x}{x}
$$

для гладких функций на промежутке $(0, \infty)$ с компактным носителем. Тогда

$$
\int_{0}^{\infty}|f(x)|^{2} \frac{d x}{x}=\frac{1}{2 \pi} \int_{-\infty}^{\infty}|\hat{f}(s)|^{2} d s .
$$

Очевидно,

$$
\widehat{f^{\prime}}(s)=(1-i s) \hat{f}(s+i)
$$

Это - простейшая ситуация, где появляются сдвиги в мнимом направлении.

2) Разностные уравнения для нескольких неоклассических семейств ортогональных многочленов, а именно многочленов Мейкснера-Поллачека, непрерывных многочленов Хана, непрерывных двойственных многочленов Хана и многочленов Вильсона, также пишутся через сдвиги в мнимом направлении (см. [1], [16]).

3) В работе автора [25] были получены некоторые элементы операционного исчисления для индексного гипергеометрического преобразования (оно также называется преобразованием Олевского или преобразованием Якоби; см. [32], [17])

$$
\begin{aligned}
g(x) & \mapsto \widehat{g}(s)= \\
= & \frac{1}{\Gamma(b+c)} \int_{0}^{\infty} g(x)_{2} F_{1}(b+i s, b-i s ; b+c ;-x) x^{b+c-1}(1+x)^{b-c} d x
\end{aligned}
$$


В [25] было показано, что индексное гипергеометрическое преобразование переводит дифференциальные операторы

$$
A g(x)=x g(x), \quad B g(x)=x \frac{\partial}{\partial x} g(x)
$$

(а поэтому и все операторы, выражающиеся полиномиально через $x, x \frac{\partial}{\partial x}$ ) в разностные операторы мнимого направления ${ }^{2}$. Существование формул (11)-(13) более-менее следует из этих утверждений. Однако этот способ получения выражений (11)-(13) также не очень прост.

4) Возникают следуюшие вопросы: возможно ли выписать явные операторы для надалгебры в случае $L^{2}$ на псевдоримановом симметрическом пространстве и для керн-представлений? можно ли это сделать по крайней мере для симметрических пространств ранга 1 ?

\section{Список литературы}

1. Andrews G.R., Askey R., Roy R. Special functions. Cambridge: Cambridge Univ. Press, 1999.

2. Van den Ban E.P., Schlichtkrull H. A residue calculus for root systems // Compositio Math. 2000. V. 123. № 1. P. 27-72.

3. Bargmann $V$. Irreducible unitary representations of the Lorentz group // Ann. Math. 1947. V. 48. P. 568-640.

4. Березин $\Phi . А$. О связи между ко- и контравариантными символами операторов на классических комплексных симметрических пространствах // ДАН СССР. 1978. Т. 241. C. $15-17$.

5. De Branges L. Tensor product spaces // J. Math. Anal. Appl. 1972. V. 38. P. 109-148.

6. Cherednik I. Harish-Chandra transform and difference operators. Preprint, available via http: //arXiv.org/abs/math/9706010.

7. Delorme P. Formule de Plancherel pour les espaces symétriques réductifs // Ann. of Math. 1998. V. 147. P. 417-452.

8. Van Dijk H., Hille S. C. Canonical representations related to hyperbolic spaces // J. Funct.Anal. 1997. V. 147. P. 109-139.

9. Гельфанд И. М., Наймарк М. И. Унитарные представления классических групп // Тр. Матем. ин-та. им. В. А. Стеклова АН СССР. 1950. Т. 36.

10. Гиндикин С. Г., Карпелевич Ф. И. Мера Планшереля для римановых симметрических пространств неположительной кривизны // ДАН СССР. 1962. Т. 145. С. 252-255.

11. Гиндикин С. Г., Карпелевич $\Phi . И$. Об одном интеграле, связанном с римановыми симметрическими пространствами неположительной кривизны // Изв. АН СССР. Сер. матем. 1966. Т. 30. С. $1147-1156$.

12. Harinck $P$. Plancherel formula pour $\mathrm{GL}(n, \mathbb{C}) / \mathrm{U}(p, q)$ // J. Reine Angew. Math. 1992 . V. 428. P. $45-95$.

13. Harinck $P$. Fonctions orbitales sur $G_{\mathbb{C}} / G_{\mathbb{R}}$. Formula d'inversion des integrales orbitales et formula de Plancherel // J. Funct. Anal. 1998. V. 153. P. 52-107.

14. Harish-Chandra. Harmonic analysis on real semisimple groups. III: The Maas-Selberg relations and the Plancherel formula // Ann. Math. 1976. V. 104. P. 117-201.

15. Хелгасон C. Группы и геометрический анализ. М.: Мир, 1987.

16. Koekoek R., Swarttouw R.F. Askey scheme of hypergeometric orthogonal polynomials and their $q$-analogues // Delft University of Technology, 1994; available via http: //aw.twi.tudelft.nl/ koekoek/research.html.

\footnotetext{
${ }^{2}$ См. также работу [6], содержащую некоторые похожие утверждения для симметрических функций в многомерном случае.
} 
17. Koornwinder T. H. Jacobi functions and analysis on noncompact symmetric spaces // Special functions: group theoretical aspects and applications / Eds. R. Askey, T.H. Koornwinder, W. Schempp. Dordrecht-Boston: D. Reidel Publ. Co, 1984. P. 1-85.

18. Макаревич Б. О. Открытые орбиты редуктивных групп в симметрических $R$-пространствах // Матем. сб. 1973. Т. 91(133). С. 390-401.

19. Молчанов $B . \Phi$. Тензорные произведения унитарных представлений трехмерной группы Лоренца // Изв. АН СССР. Сер. матем. 1979. Т. 43. № 4. С. 860-891.

20. Молчанов В. Ф. Формула Планшереля для гиперболоидов // Тр. Матем. ин-та им. В. А. Стеклова АН СССР. 1980. Т. 147. С. 65-85.

21. Молчанов В.Ф. Формула Планшереля для псевдоримановых симметрических пространств ранга 1 // ДАН СССР. 1986. Т. 290. № 3. С. 545-549.

22. Молчанов $B . \Phi$. Гармонический анализ на однородных пространствах // Совр. пробл. математики. Фундаментальные направления. Некоммутативный гармонический анализ-II. Т. 59. М.: ВИНИТИ, 1990. С. 5-144.

23. Неретин Ю. А. Псевдоримановы симметрические пространства: однотипные реализации и открытые вложения в грассманианы // Записки научн. семин. ПОМИ РАН. Т. 256. C. $145-167$.

24. Neretin Yu. A. Plancherel formula for Berezin deformation of $L^{2}$ on Riemannian symmetric space // J. Funct. Anal. 2001. V. 189. P. 336-408.

25. Неретин Ю. А. Индексное гипергеометрическое преобразование и имитация анализа ядер Березина на гиперболических пространствах // Матем. сб. 2001. Т. 192 . №3. C. $83-114$.

26. Neretin Yu. A. Matrix balls, radial analysis of Berezin kernels and hypergeometric determinants // Moscow Math. J. 2001. V. 1. P. 157-220.

27. Pukanszky L. On the Kronecker products of irreducible unitary representations of the $2 \times 2$ real unimodular group // Trans. Amer. Math. Soc. 1961. V. 100. P. 116-152.

28. Rosengren $H$. Multilinear Hankel forms of higher order and orthogonal polynomials // Math. Scand. 1998. V. 82. № 1. P. 53-88.

29. Rosengren $H$. Multivariable orthogonal polynomials and coupling coefficients for discrete series representations // SIAM J. Math.Anal. 1999. V. 30. P. 233-272.

30. Unterberger A., Upmeier $H$. The Berezin transform and invariant differential operators // Comm. Math. Phys. 1994. V. 164. P. 563-597.

31. Вершик А.М., Гельфанд И. М., Граев М.И. Представления $\mathrm{SL}_{2}(R)$, где $R$ - кольцо функций // УМН. 1973. Т. 28. № 5. С. 83-128.

32. Weyl H. Über gewonliche lineare Differentialgleichungen mis singularen Stellen und ihre Eigenfunktionen 2 Note // Nachr. Konig. Gess. Wissen. Gottingen. Math.-Phys, 1910. P. 442-467; Перепечатано в книге Weyl H., Gessamelte Abhandlungen. Bd. 1. Springer, 1968. P. 224-247.

Институт теоретической и экспериментальной физики

E-mail: neretin@ main.mccme.rssi.ru

Поступило в редакцию 6.IV.2001 